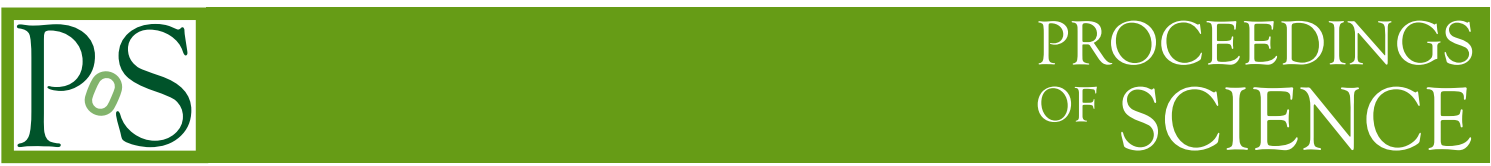

\title{
Hard X-ray Telescopes onboard ASTRO-H
}

\author{
Hideyuki Mori $^{* a}$, Hironori Matsumoto ${ }^{a}$, Hideyo Kunieda ${ }^{a b}$, Takuya Miyazawa $^{b}$, Naoki \\ Ishida $^{b c}$, Akio Suzuki ${ }^{c}$, Hiroyuki Furuta $^{c}$, Yoshihiro Yamamoto $^{c}$, Hiroyoshi Kato ${ }^{b}$, \\ Yusuke Miyata $^{b}$, Tadatsugu Demoto $^{b}$, Tsuyoshi Watanabe ${ }^{b}$, Yuji Kuroda ${ }^{b}$, Shunya \\ Takizawa $^{b}$, Toshihiro Iwase ${ }^{b}$, Shigetaka Saji ${ }^{b}$, Sasagu Tachibana ${ }^{b}$, Yasunori \\ Babazaki $^{b}$, Tatsuya Hibino ${ }^{b}$, Ren Nakamichi ${ }^{b}$, Keisuke Tamura ${ }^{b}$, Kazunori Ishibashi \\ ${ }^{b}$, Yuzuru Tawara ${ }^{b}$, Hisamitsu Awaki $^{d}$, Satoshi Sugita ${ }^{d}$, Daichi Kurihara ${ }^{d}$, Kentaro \\ Uesugi $^{e}$, Yoshio Suzuki ${ }^{e}$, and the ASTRO-H HXT team \\ ${ }^{a}$ Kobayashi-Maskawa Institute for the Origin of Particles and the Universe (KMI), Nagoya \\ University, Furo-cho, Chikusa-ku, Nagoya, 464-8602, Japan \\ ${ }^{b}$ Division of Particle and Astrophysical Science, Graduate School of Science, Nagoya University, \\ Furo-cho, Chikusa-ku, Nagoya, 464-8602, Japan \\ ${ }^{c}$ Tamagawa Engineering Co. Ltd., 1218-18 Azajifuku, Kagiya-cho, Kasugai, Aichi, 480-0304, \\ Japan \\ ${ }^{d}$ Department of Physics, Graduate School of Science and Engineering, Ehime University, \\ Bunkyo-cho, Matsuyama, Ehime, 790-8577, Japan \\ e JASRI/SPring-8, Sayo-cho, Syo, Hyogo, 679-5198, Japan \\ E-mail: morieu.phys.nagoya-u.ac.jp
}

\begin{abstract}
We develop two Hard X-ray Telescopes (HXT) onboard ASTRO-H, the 6th Japanese X-ray satellite to be launched in 2015. The HXT, together with Hard X-ray Imager, enables us to collect $\mathrm{X}$-rays up to $70 \mathrm{keV}$ for imaging spectroscopy. We completed the fabrication of two HXTs in July 2013. Moreover, the ground calibration of the HXTs has been done at the synchrotron radiation facility, SPring-8. The HXT angular resolution was found to be 1.9 arcmin (Half Power Diameter) at $30 \mathrm{keV}$. In addition, the effective areas of $170 \mathrm{~cm}^{2}$ at $30 \mathrm{keV}$ and $82 \mathrm{~cm}^{2}$ at $50 \mathrm{keV}$, which satisfy the requirement, were obtained. In this presentation, we also report the detailed $\mathrm{X}$-ray performance of the HXT.
\end{abstract}

KMI International Symposium 2013 on" Quest for the Origin of Particles and the Universe", 11-13 December, 2013

Nagoya University, Japan

\footnotetext{
* Speaker.
} 


\section{Introduction}

ASTRO-H [四, the 6th Japanese-US collaborated mission, has characteristics of the unprecedented high energy resolution achieved by X-ray microcalorimeter and the imaging capability up to $70 \mathrm{keV}$ due to the Hard X-ray Telescopes (HXTs) [2] and the Hard X-ray Imager (HXI) [B]]. Scientific objectives utilizing the wide-band imaging spectroscopy of the HXT+HXI are as follows: (1) to detect supermassive black holes buried in optically-thick dusty tori with absorption column densities $\left(N_{\mathrm{H}}\right)$ of $>10^{24} \mathrm{~cm}^{-2}$, (2) to detect non-thermal emission caused by particle acceleration from some clusters of galaxies, etc.. To achieve these scientific goals, the angular resolution and effective area are required to be 1.7 and $150 \mathrm{~cm}^{2}$ at $30 \mathrm{keV}$ and $55 \mathrm{~cm}^{2}$ at $50 \mathrm{keV}$, respectively.

The HXT is composed of the mirror module, pre-collimator, and two thermal shields. The mirror module adopts the conical approximation of the Wolter-I type grazing incident optics and nested thin-foil structure to enhance their effective areas. In the mirror modules, 213 pairs of primary and secondary mirrors are arranged concentrically with alignment bars, by which we can tune the radial positions of the mirrors. Since the HXT is divided into three segments, the total number of the mirror shells amounts to 1278 per telescope. The reflector coated on the mirror surface is depth-graded $\mathrm{Pt} / \mathrm{C}$ multilayer, which give high $\mathrm{X}$-ray reflectivity with total reflection and Bragg reflection. The pre-collimator and thermal shields (PET films with $5 \mu \mathrm{m}$ thickness) are attached to reduce stray lights and to control the HXT temperature $\left(22{ }^{\circ} \mathrm{C}\right.$ in orbit), respectively. A

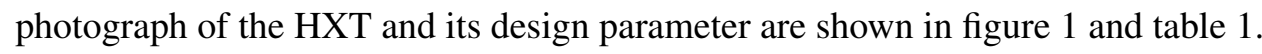

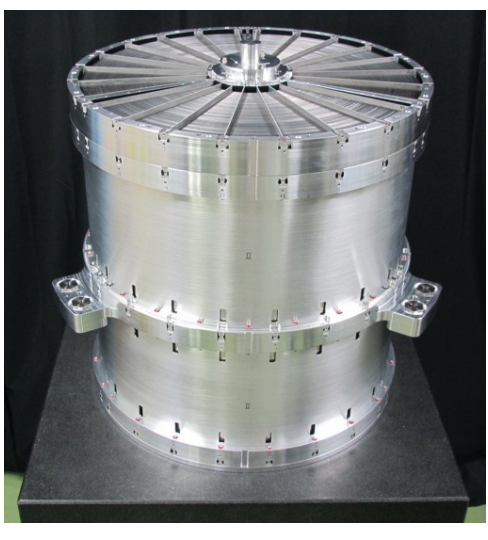

Figure 1: Photograph of the HXT-1

\begin{tabular}{lc}
\hline \hline Focal length & $12 \mathrm{~m}$ \\
Material of mirror substrates & Aluminum \\
Axial length/Thickness of substrates & $200 \mathrm{~mm} / 0.2 \mathrm{~mm}$ \\
Material of reflectors & $\mathrm{Pt} / \mathrm{C}$ multilayer \\
Number of shells & 213 \\
Diameters of the innermost shell & $120 \mathrm{~mm}$ \\
Diameters of the outermost shell & $450 \mathrm{~mm}$ \\
Incident angles & $0.07-0.27$ \\
Weight per telescope & $62 \mathrm{~kg}$ \\
\hline \hline
\end{tabular}

Table 1: Design parameters of the HXTs

\section{SPring-8 BL20B2 beamline}

SPring- 8 is the 3 rd-generation synchrotron radiation facility located at Hyogo Pref. We used the BL20B2 beamline to improve the imaging quality in the mirror assembly and to measure the Xray performance in the $20-70 \mathrm{keV}$ band for the HXT ground calibration [ [4]. The schematic view of the measurement configuration is shown in figure $\square$. Both the HXT and the detector (Image Intensifier C7336 + optical CCD camera C4480, HAMAMATSU Photonics K.K.) are mounted on the respective stages. These stages move in the horizontal $(\mathrm{Y})$ and vertical $(\mathrm{Z})$ directions perpendicular to incident $\mathrm{X}$-rays. In addition, the telescope stage rotates around the $\mathrm{X} / \mathrm{Y} / \mathrm{Z}$ axes.

We can utilize X-rays monochromatized by Si Double Crystal Monochrometer $\left(E / \Delta E \sim 10^{4}\right)$ with a typical flux of $\sim 10^{7}$ photons $\mathrm{s}^{-1} \mathrm{~mm}^{-2}$. The beam intensity is quite stable; a typical beam 
fluctuation is $10^{-3}$. A movable slit is placed between the transport duct and the HXT stage to change the beam size we want. The X-ray beam collimated to $10 \mathrm{~mm} \times 10 \mathrm{~mm}$ size is generally used in the measurement to ensure highly parallelism of $\sim 10^{\prime \prime}$. In order to illuminate the full aperture of the HXT by this small beam, we move the stages mounting the HXT and the detector synchronously in the Y/Z directions. The number of the pointings is 522 per one segment. X-ray snapshots obtained at each pointing are summed together to reconstruct a focal plane image of the HXT. The focal plane image taken at 30 and $50 \mathrm{keV}$ are shown in figure 3 .

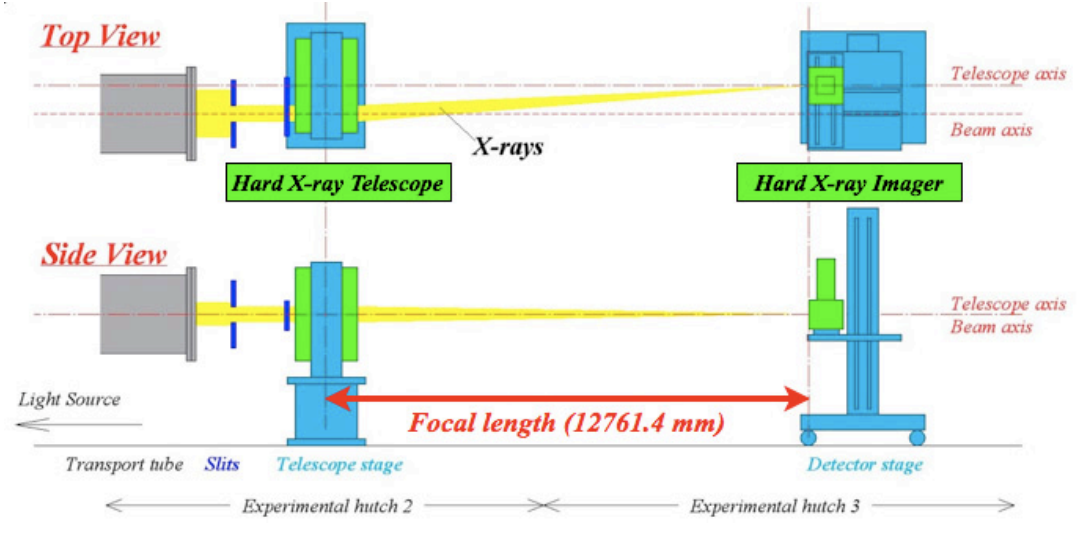

Figure 2: Schematic top and side views of the configuration in the BL20B2

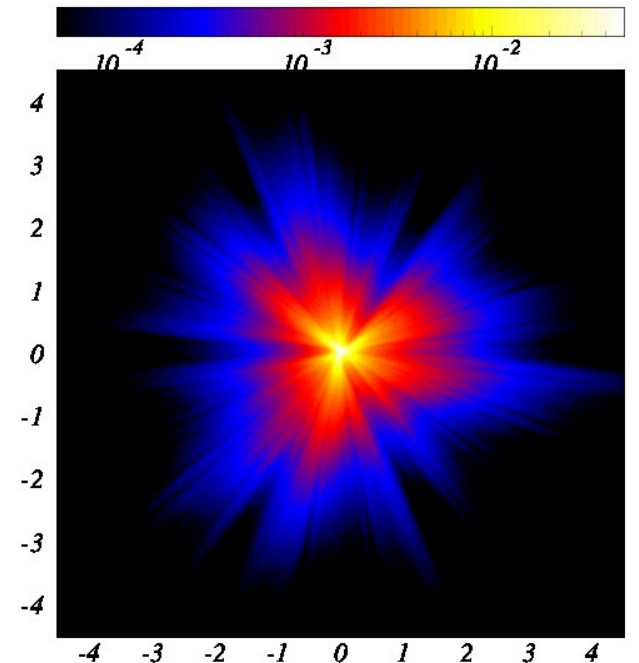

XIS raster_dkcorr_fcorr_hcut_segall_30keV_tele_axis_hxi-fov,fit:

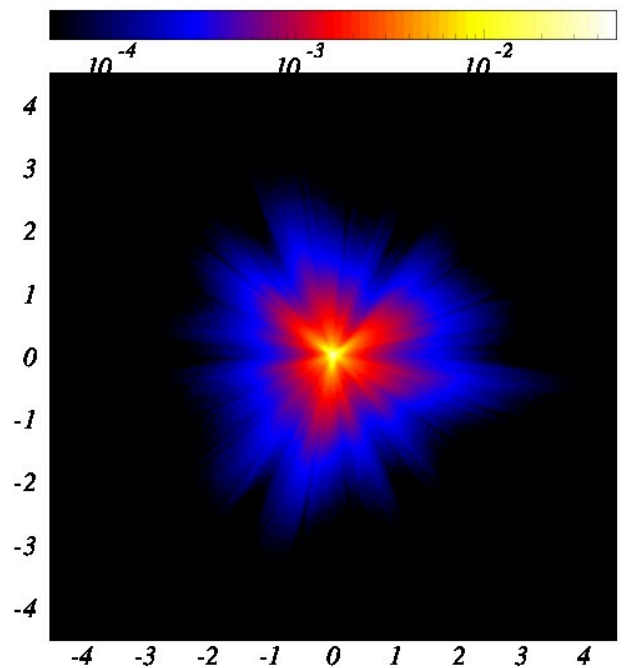

XIS raster_dkcorr_fcorr_hcut_segall_50keV_tele_axis_hxi-fov,fit

Figure 3: Focal plane images of the HXT-2 taken at 30 (left) and 50 (right) keV. The image size is 9!.2 $\times 9 ! 2$ consistent with the HXI field of view. The color scale represents surface brightness normalized by the total X-ray flux.

\section{X-ray performance of the HXTs}

Using the focal plane images described above, we evaluated the HXT performance in terms of (1) an angular resolution and (2) an effective area. The angular resolution and effective area measured at some representative energies are summarized in table $\square$. Here, the angular resolution is given by Half Power Diameter (HPD), within which half of the focused X-rays is accumulated. Since a typical systematic error of the angular resolution is 0.1 , the two HXTs almost meet the 


\begin{tabular}{|c|c|c|c|c|c|c|}
\hline Energy $(\mathrm{keV})$ & 20 & 30 & 40 & 50 & 60 & 70 \\
\hline \multicolumn{7}{|c|}{ Angular resolution (Note: typical systematic error is $\sim 0.1$ ) } \\
\hline HXT-1 & - & $1 ! 92$ & $1 ! 94$ & $1 ! 80$ & $1 ! 67$ & 1.49 \\
\hline HXT-2 & 1.92 & $1 ! 88$ & 1.90 & 1,78 & 1.70 & 1.54 \\
\hline \multicolumn{7}{|c|}{ Effective area $\left(\mathrm{cm}^{2}\right)$} \\
\hline HXT-1 & - & $170 \pm 2$ & $123 \pm 1$ & $82 \pm 2$ & $50 \pm 1$ & $24.5 \pm 0.4$ \\
\hline HXT-2 & $288 \pm 3$ & $178 \pm 1$ & $125 \pm 1$ & $82 \pm 0.4$ & $49.2 \pm 0.3$ & $24.8 \pm 0.4$ \\
\hline
\end{tabular}

Table 2: Summary of the angular resolution and effective area of the HXTs
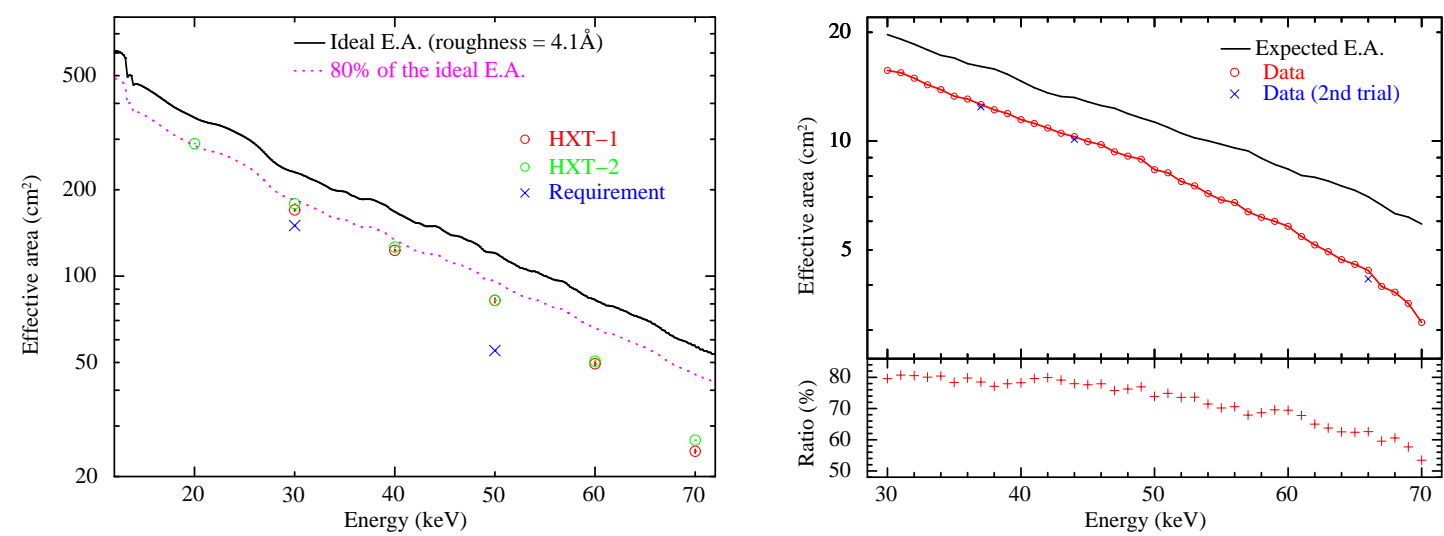

Figure 4: Energy dependence of the effective areas for the HXTs (left) and the limited aperture (right).

requirement for the imaging quality. Since the inner mirror shells have better imaging quality than the outer ones, the HPD has a significant energy dependence ( 1.9 at $30 \mathrm{keV}$ and 1.5 at $70 \mathrm{keV})$.

Figure 9 (left) shows the HXT effective area (hereafter EA) as a function of X-ray energies. The measured EA is larger than the requirement by $13 \%$ at $30 \mathrm{keV}$ and by $60 \%$ at $50 \mathrm{keV}$. In this panel, we indicated the expected EAs (black solid curve), assuming the interfacial roughness of the $\mathrm{Pt} / \mathrm{C}$ multilayer of $4.1 \AA$. In the $20-40 \mathrm{keV}$ band, the measured EAs were $80 \%$ of the calculation (magenta dotted line). However, the ratio of the measured EA to the calculation declined to be $50 \%$ at $70 \mathrm{keV}$. Although the examination on this degradation is still on going, X-ray scattering due to the mirror distortion or deviations from the assumed roughness may be the cause. In order to investigate more detailed energy dependence, we measured the effective area for a limited aperture in the $20-70 \mathrm{keV}$ band with a $1 \mathrm{keV}$ pitch, consistent with the HXI energy resolution. The result is plotted in the right panel of figure $\mathrm{H}$. At 37,44 , and $66 \mathrm{keV}$, we measured twice; the systematic uncertainty of this measurement was found to be $1-5 \%$. In the lower column of this panel, the ratios to the expected EA are also indicated.

\section{References}

[1] Takahashi, T., et al., The ASTRO-H Mission, Proc. SPIE, 7732, pp. 77320Z-77320Z-18

[2] Kunieda, H., et al., Hard X-ray Telescope to be onboard ASTRO-H, Proc. SPIE, 7732

[3] Kokubun, M., et al., Hard X-ray Imager (HXI) for the ASTRO-H Mission, Proc. SPIE, 7732

[4] Miyazawa, T., et al., Current status of hard x-ray characterization of ASTRO-H HXT at SPring-8, Proc. SPIE, $\mathbf{7 7 3 2}$ 